\title{
Biomarkers in the Diagnosis of Heart Failure in Dogs
}

\author{
Lyudmila Annikova* \\ Saratov State Agrarian University named after N.I. Vavilov, Saratov, 410012, 1 Teatralnaya \\ Ploshchad, Russian Federation
}

\begin{abstract}
Every doctor, one way or another, is faced with the problem of chronic heart failure (CHF) in animals [1]. Over 3 million dogs suffer from some form of acquired heart disease. In recent years, biomarkers, mainly cardiac troponin and natriuretic peptides, have become the main source of information for both diagnosis and monitoring of the human heart. Today, the need for diagnostics based on blood samples has become evident also when working with dogs and cats.
\end{abstract}

\section{Introduction}

In recent years, many developments have taken place in the field of clinical veterinary medicine. Achievements of humane medicine are already successfully used in almost all areas of veterinary medicine, and veterinary cardiology is no exception.

Heart failure manifests itself in the inability of the heart to pump blood at a sufficient rate for the body. When the heart tries to work more efficiently, the damage worsens. For a biomarker to be applicable in clinical practice, it is necessary that its amount changes in proportion to the development of certain processes that characterize the disease, and thus give an idea of the presence, severity and prognosis of the disease. Ideally, the biomarker shall be stable and easily measurable with readily available, fast and inexpensive methods. Biomarkers are used in veterinary medicine to assess organ function: for example, the content of carbamide and creatinine is indicative for monitoring the state of the kidneys, and alanine aminotransferase - for assessing liver cell damage. Previously, conventional assays for enzymes (such as creatine kinase) used in canine heart disease have not shown the sensitivity and specificity sufficient for successful clinical use. However, tests for cardiac troponin and natriuretic peptides have proven to be important sources of information in the case of canine heart disease. This review examines the possibilities of using these samples in veterinary medicine and obtaining information with their help [2].

\section{Methods}

The aim of our studies was to determine the suitability of the commercial Triage BNP kit for use in veterinary practice for the detection of heart failure in dogs.

\footnotetext{
* Corresponding author: alaw67@inbox.ru
} 
The main biomarkers defining heart disease are creatine phosphokinase, cardiac troponin I, and type B natriuretic peptide. CPK is found in the greatest quantities in the cells of skeletal muscles, brain and smooth muscles. There are 3 isoenzymes: CPK-MB cardiac type, CPK-MM - muscle type, CPK-BB - brain type. CPK is mainly used in the diagnosis of myocardial infarction or skeletal muscle injury.

Historically, for the diagnosis of HF, it has been (and continues to be used) to determine the activity of one of the components of CPK - the CF-fraction of creatine kinase (CPKMB). An increase in CPK-MB activity is most specific for MI. With MI, the increase in CPK-MB activity begins within 4-8 hours after an acute attack and reaches a maximum in 12-24 hours; on the third day, the activity of this fraction returns to normal. With the expansion of the zone of myocardial ischemia, the activity of CPK-MB remains high for much longer, which makes it possible to diagnose myocardial infarction in more distant periods of observation.

\section{Results}

The presence of CPK-MB in serum, however, is not in $100 \%$ of cases specific for myocardial damage, since $\mathrm{CPK}$ is also increased in patients with damage to skeletal muscles. CPK can increase, for example, after intramuscular administration of drugs or after increased and prolonged muscle activity. High CPK activity is observed with hypoxia of skeletal muscles, with shock or embolism of the arteries. However, arrhythmia, tachycardia and heart failure practically do not affect the level of CPK-MB [5-6].

An invaluable contribution to the solution of all of the above issues was introduction into clinical practice for the determination of myocardial markers in the serum (plasma) of blood. The troponin complex consists of three subunits (cTnl, cTnTn, and cTnC) that regulate the excitation-contraction process in the muscle cells of the heart. cTnl is an inhibition component that prevents the interaction between actin and myosin until cTnC binds calcium ions. Damage to the sarcomere leads to the cleavage of cTnl from actin and subsequent destruction of the cell membrane. Therefore, cTnl enters the extracellular space and biological fluids. Therefore, a high level of cTnl in serum or plasma can be regarded as a very sensitive and specific indicator of myocardial cell damage and subsequent necrosis. The similar structure of cTnl in mammals allows accurate measurements in dogs and cats using immunological assays developed for humans.

In human medicine, the content of cardiac troponins is a general diagnostic criterion for acute coronary syndromes. An increase in the level of cTnl is recorded within 3-4 hours after myocardial injury and remains high for 4-7 days after myocardial infarction. In people with chronic heart failure, the level of cTnl remains moderately elevated, therefore, it can be used to monitor the development of the disease and is a source of prognostic information. An elevated level of cTnl provides a basis for poor prognosis and is an independent predictor of deaths [7-8]. It is likely that cTnl levels have the same predictive power in animals, although myocardial infarction is significantly less common in dogs.

An invaluable contribution to the solution of all the above issues was the introduction into clinical practice for the determination of myocardial markers in the blood serum (plasma) - troponins I and T - absolutely specific and highly sensitive for the situation with ACS. Currently, the leading cardiological communities in Europe and the United States have adopted recommendations for the diagnosis of the main forms of ACS, in which the determination of the troponin $\mathrm{T}$ concentration (in serum, plasma) of blood is considered as the most important diagnostic criterion for MI.

A number of studies have shown the effectiveness of the test for the level of cardiac troponin I in dogs [8]. Cardiac troponin I is a marker that is not specific for its underlying causes. This means that an increase in the level of cTnl can be caused both primarily by 
heart disease and by systemic diseases that affect the myocardium secondarily. According to human medicine, an increase in cTnl levels occurs at the final stage of renal failure, with sepsis and various injuries. In dogs, the spike in troponin I levels can be caused by pyometra, gastrectasia, pericardial effusion, trauma, and sepsis. Myocarditis leads to an increase in cTnl levels hundreds of times, as is the case in dogs with Chagas disease and babesiosis.

Cardiac troponin analysis can be used in conjunction with other diagnostic methods to provide reliable information in cases of sepsis and gastrectasia. In patients with severe arrhythmia or systolic dysfunction, a significant increase in cTnl is associated with myocarditis and can be used to assess the effectiveness of treatment. In a small pilot study, Linklater et al showed shorter survival times in dogs with mitral valve disease and elevated cTnl levels admitted to the hospital for emergency treatment for congestive heart failure. Further study is aimed at obtaining more detailed information on the possibilities of using cTnl data in the diagnosis of congenital and acquired heart diseases.

Brain natriuretic peptide (NTproBNP) has become another reliable cardiomarker in recent years. It is reliably, strictly and evidence-based that the detection of its increased concentrations indicates the presence of heart failure (HF) in the patient. Therefore, in the diagnosis of HF all over the world, the determination of the level of this peptide has already become the standard. It shall be noted that a number of large international studies have shown that use of the NT-proBNP and BNP tests in clinical practice can reduce the cost of examining patients and accurately predict the presence or absence of heart failure, even before echocardiography. Another important fact: at present, the test is widely used not only in cardiovascular pathology, but also in some clinical conditions, accompanied by a decrease in the contractility of the heart muscle [6]. With violations of heart function, constant overstimulation of the renin-angiotensin system occurs, which leads to an enlargement of the heart, volume overload and, as a result, to coronary heart disease. Natriuretics resist these processes by stimulating natriuresis, renal blood flow, diuresis and vasodilation, and also positively affect diastolic function. Atrial natriuretic peptide (ANP) and B-type natriuretic peptide (BNP) levels increase mainly in response to increasing pressure on the myocardial wall. ANP is synthesized primarily in the atria, while BNP is secreted by both atrial and ventricular muscle cells. Both peptides are precursor molecules that are subsequently cleaved by a proteolytic enzyme into the same number of active $\mathrm{C}$ fragments (often referred to as C-ANP and C-BNP) and inactive N-fragments (NT-proANP and NT-proBNP). C-ANP and C-BNP have an ultra-short half-life and are therefore difficult to measure in blood. NT-proANP and NT-proBNP have a longer half-life, are more stable and available in samples, therefore, they are applicable in clinical trials. Today there are NT-proANP and NT-proBNP ELISA tests specifically for dogs.

Today, the measurement of the content of natriuretic peptides in serum and plasma is considered an important analysis in the monitoring of human heart disease. For emergency care, NT-proBNP can be used in conjunction with a physical examination, chest radiography, and electrocardiography to distinguish the effects of dyspnea from coronary artery disease. By itself, the BNP level is an indicator of the risk of death and heart failure in asymptomatic patients, making it possible to examine such patients and reliably assess the severity of their condition. Based on the BNP content, the short-term effect of antiischemic therapy can also be assessed. When interpreting the results of the analysis, it shall be borne in mind that in a person the level of natriuretics in the blood changes depending on the characteristics of the functioning of the kidneys, gender, weight and age. Several studies have recently been published evaluating the efficacy of applying natriuretic peptide measurements, namely NT-proBNP, to canine patients. 
To solve the set tasks, we selected 2 groups of animals. Group I consisted of 5 dogs, from 7 to 9 years old, with signs of heart failure of various classes (see Appendix 1 for the classification of HF).

Group II consisted of 4 dogs, aged from 7 to 9 years. The animals were clinically healthy.

All animals underwent physical examinations and special examinations, namely: electrocardiography, X-ray examinations, laboratory diagnostics.

In laboratory diagnostics, the following was checked: a general blood test, a biochemical blood test, and the concentration of cardiac biomarkers (CPK, NP) was determined.

The content of B-natriuretic peptide in whole blood samples was determined with a specially designed Triage BNP test panel. The Triage BNP test is a disposable panel designed for immunofluorescence analysis to determine the concentration of B-natriuretic peptide. Previously, all dogs were sampled venous blood in special tubes treated with the anticoagulant EDTA (Figures 1 and 2).

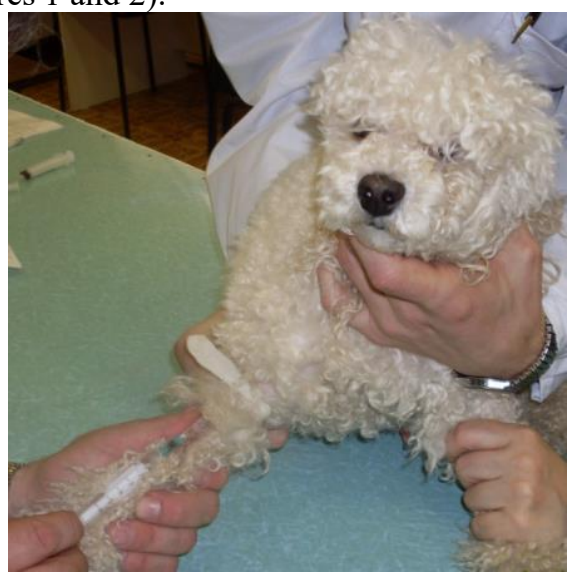

Fig. 1. Blood sampling from a poodle at the Department of Obstetrics, Surgery and Animal Therapy.

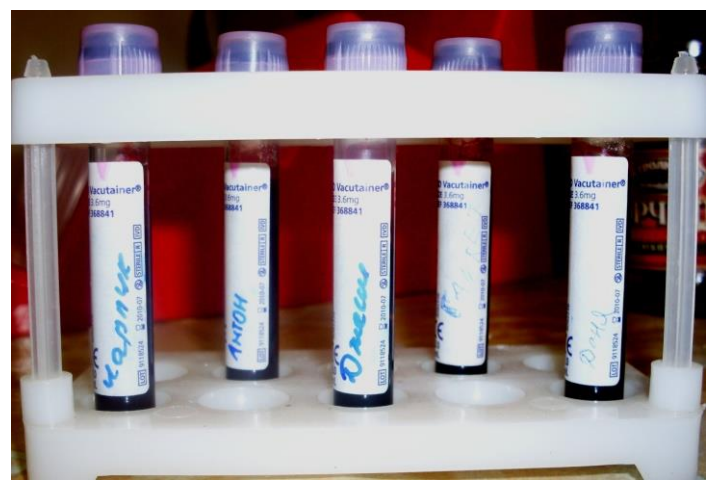

Fig. 2. Blood tubes treated with EDTA anticoagulant.

Blood samples from the EDTA tube were shaken and applied to a special area of the panel using a pipette designed to transfer an appropriate amount of sample $(250 \mathrm{ml})$ to the panel (Fig. 3). The blood test was carried out immediately after taking it from the animal. 


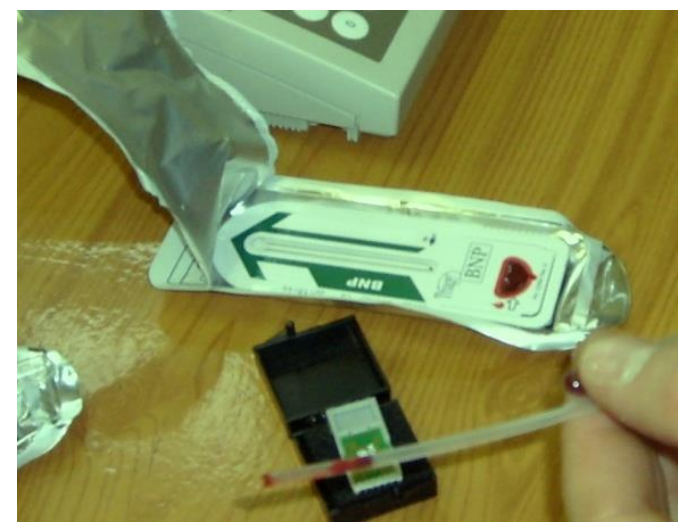

Fig. 3. Disposable Triage BNP Test Panel.

After applying the blood sample, the diagnostic device was inserted into the Triage analyzer (Fig. 4), preprogrammed to automatically conduct B-NP analysis after the sample reacted with the reagents on the diagnostic device. The entire process of carrying out one test - from applying the sample to obtaining the result - took 15 minutes. Substance sampled concentration analysis was performed based on the amount of fluorescence detected by the analyzer in a dedicated measurement area of the panel. The Triage BNP Test panel contained the complete set of reagents required for the test (stabilizers, fluorescently labeled mouse mono- and polyclonal antibodies against B-natriuretic peptide).

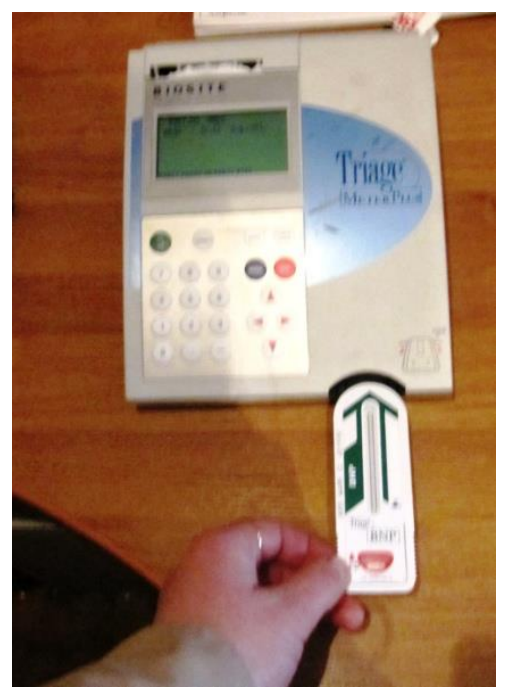

Fig. 4. Conducting the BNP analysis.

The results of the Triage B-NP test were evaluated in conjunction with all the obtained clinical and laboratory data. Evaluation of the effectiveness of this test was carried out on the condition that the object of the study were whole venous blood samples treated with EDTA as an anticoagulant. When examining other forms of samples, other methods of drawing blood and other anticoagulants, the effectiveness of the test could not be evaluated.

It was possible that certain factors, such as technical or procedural errors, as well as presence of additional substances (not mentioned below) in blood products, could interfere with the validity of testing and contribute to erroneous results.

The studies were carried out from September 2019 to March 2020 at the Department of Animal Diseases and Veterinary-Sanitary Inspection, SSAU named after N.I. Vavilov and at the veterinary 
clinic of Dr. Annikov. We clinically studied 128 dogs of various breeds and ages, of which 5 animals with signs of heart failure of varying degrees were identified, and 4 clinically healthy animals of the corresponding breeds and ages were selected.

All 9 dogs (Table 1) underwent a study of whole blood samples with the commercial Triage BNP test kit, as well as physical examinations, recording and decoding of electrocardiograms (Fig. 5a, b), general, biochemical blood tests, and X-ray images of organs of the chest.

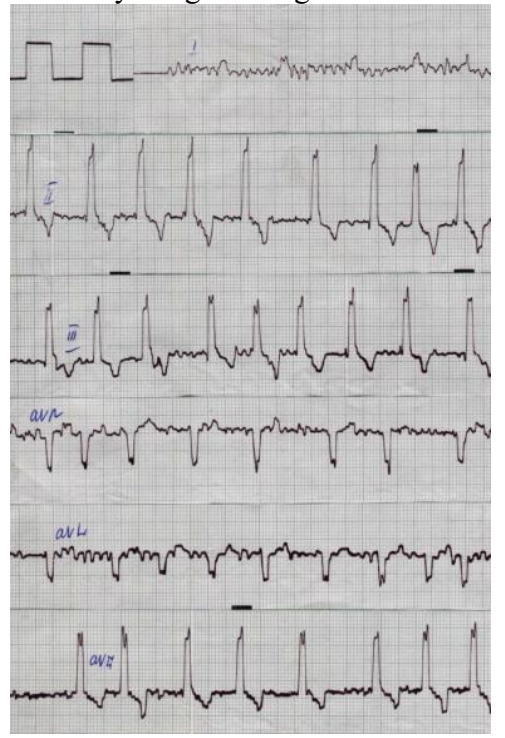

Fig. 5. Electrocardiogram of a dog, + , dachshund, 9 years old, with signs of HF III FC.

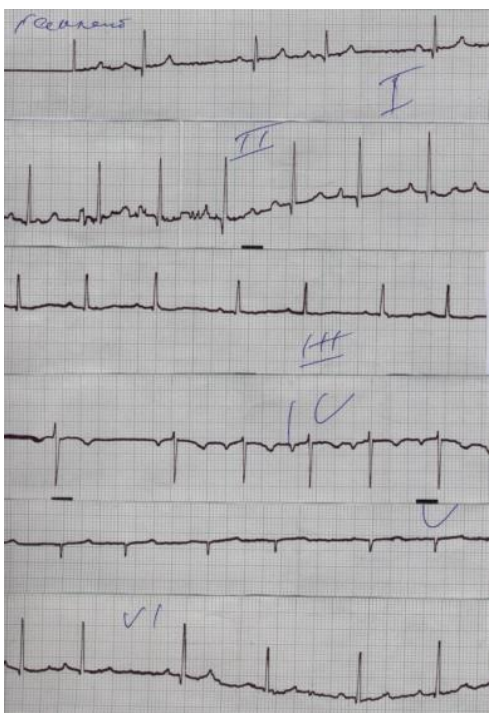

Fig. 6. Electrocardiogram of a dog, $\hat{\partial}$, dachshund, 8 years old, clinically healthy animal.

Table 1. Information about the investigated animals.

\begin{tabular}{|l|c|c|}
\hline \multicolumn{1}{|c|}{ Dog breeds } & $\begin{array}{c}\text { Experienced group of dogs } \\
\text { with HF, heads }\end{array}$ & $\begin{array}{c}\text { Control group of clinically } \\
\text { healthy dogs, heads }\end{array}$ \\
\hline Poodle & 1 & 1 \\
\hline Dachshund & 2 & 2 \\
\hline Pekingese & 2 & 1 \\
\hline
\end{tabular}




\begin{tabular}{|l|l|c|}
\hline Total & 5 & 4 \\
\hline
\end{tabular}

The dogs in the experimental group had varying degrees of HF (Table 2).

Table 2. Distribution of dogs by functional classes of (FC) HF.

\begin{tabular}{|c|c|c|c|c|}
\hline FC & I & II & III & IV \\
\hline $\begin{array}{c}\text { number, } \\
\text { heads }\end{array}$ & 0 & 3 & 2 & 0 \\
\hline
\end{tabular}

Animals with II degree of HF (poodle, 7 years old; dachshund, 7 years old, Pekingese, 8 years old) had mild symptoms, which were expressed by fatigue with moderate physical and emotional stress, sometimes there was a cough.

Dogs with III degree HF (Dachshund, 9 years old; Pekingese, 8 years old) with minor physical exertion showed shortness of breath, fatigue, cough, mainly at night.

In healthy animals (poodle, 8 years old; dachshund, 8 years old; dachshund, 7 years old; Pekingese, 7 years old), respiratory arrhythmia was observed, the animals were active.

Data on animal studies are presented in Table 3.

Table 3. Basic indicators of physical and special studies.

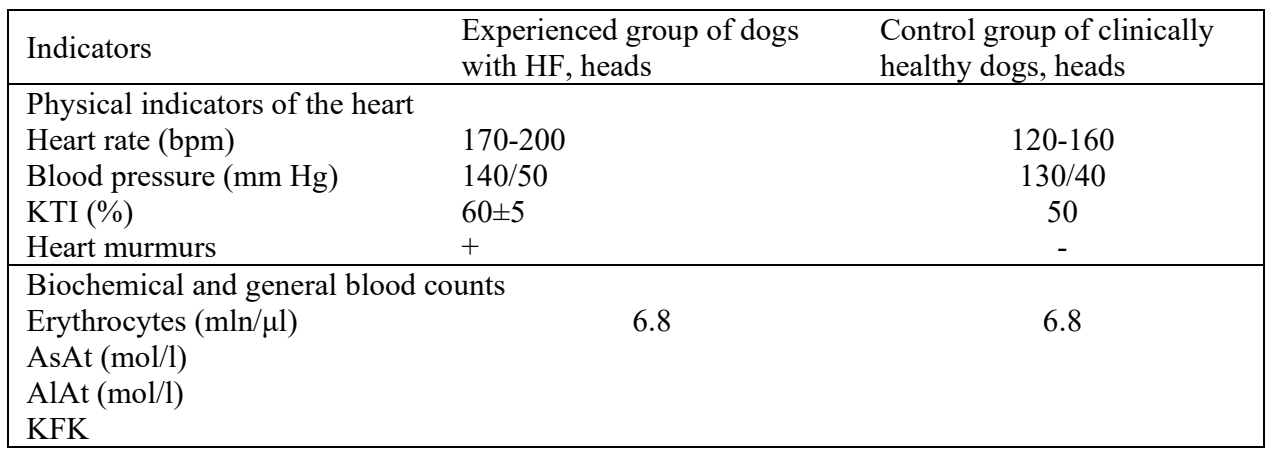

\section{Discussion}

From the above indicators, we did not find reliable signs that distinguish sick animals from healthy ones, especially if it is considered that presence of cough and shortness of breath indicate not only heart failure, but can also be signs of respiratory diseases. ECG indicators can serve in favor of HF. In dogs with heart failure, the signs of hypertrophy of the heart chambers were clearly expressed on electrocardiograms, sinus arrhythmias with the presence of extrasystoles were often observed. ST segment change and T wave depression.

In the study of natriuretic peptide with the commercial Triage BNP test kit in all dogs, regardless of the severity of the level of heart failure, the BNP level was below the sensitivity threshold of this device and did not exceed $5 \mathrm{pg} / \mathrm{ml}$.

\section{Conclusion}

Heart failure refers to those diseases in the diagnosis of which not one, even a proven method, is important, but a set of diagnostic methods. The significance of symptoms and clinical signs is extremely high, since it is they that make the cardiologist suspect that the patient has heart failure syndrome. Therefore, examination of a patient with heart failure 
syndrome always begins with a conversation between a cardiologist and a patient. The problem, however, is that the symptoms of heart failure, especially those that the patient himself/herself may notice, are not unique to this disease. In addition, with different forms of heart failure (left ventricular and right ventricular), the signs may be different, they may not coincide in different animals. For example, despite the fact that shortness of breath, swelling of the ankles and rapid fatigue are considered to be the characteristic symptoms of heart failure.

Use of the commercial Triage BNP test kit of reagents for determining BNP is not suitable in veterinary practice due to their insufficient sensitivity (the range of BNP values in dogs is much lower than in humans).

\section{References}

1. L. Adolph, R. Lorenz, Enzymdiagnostik bei Herz-, Leber- und Pankreaserkrankungen (1978)

2. J. Healey, R. Davies, S. Smith, Can J. Cardiol, 19, 4 (2003)

3. W. Peacock, M. DeMarco, M. Fonarow, NEnglJMed, 358 (2008)

4. K. Schober, B. Kirbach, G. Oechtering, J. Vet Cardiol, 1 (1999)

5. M. Sleeper, C. Clifford, L. Laster, J. Vet Intern Med, 15, 5 (2001)

6. A. Linklater, M. Lichtenberger, D. Thamm, J. Vet Emerg Crit Care, 17, 3 (2007)

7. K. Swedberg, J. Cleland, H. Dargie, Eur Heart J., 26 (2005)

8. A. Maisel, V. Bhalla, E. Braunwald, Nat Clin Pract Cardiovasc Med, 3, 1 (2006) 